\title{
Forced oscillation of higher-order nonlinear neutral difference equations with positive and negative coefficients
}

\author{
Yali Gao, Yuangong Sun*, Bin Zha and Hongshuang Liu
}

"Correspondence:

sunyuangong@yahoo.cn

School of Mathematical Sciences,

University of Jinan, Jinan, Shandong

250022, China

\section{Springer}

\begin{abstract}
In this paper, we study the forced oscillation of the higher-order nonlinear difference equation of the form

$$
\Delta^{m}[x(n)-p(n) x(n-\tau)]+q_{1}(n) \Phi_{\alpha}\left(n-\sigma_{1}\right)+q_{2}(n) \Phi_{\beta}\left(n-\sigma_{2}\right)=f(n),
$$

where $m \geq 1, \tau, \sigma_{1}$ and $\sigma_{2}$ are integers, $0<\alpha<1<\beta$ are constants, $\Phi_{*}(u)=|u|^{*-1} u$, $p(n), q_{1}(n), q_{2}(n)$ and $f(n)$ are real sequences with $p(n)>0$. By taking all possible values of $\tau, \sigma_{1}$ and $\sigma_{2}$ into consideration, we establish some new oscillation criteria for the above equation in two cases: (i) $q_{1}=q_{1}(n) \leq 0, q_{2}=q_{2}$ (n) $>0$; (ii) $q_{1} \geq 0, q_{2}<0$.

MSC: $39 \mathrm{~A} 10$
\end{abstract}

Keywords: forced oscillation; neutral difference equation; positive and negative coefficients; higher-order

\section{Introduction}

Qualitative theory of difference equations has received much attention in recent years due to its extensive applications in computer, probability theory, queuing problems, statistical problems, stochastic time series, combinatorial analysis, number theory, electrical networks, genetics in biology, economics, psychology, sociology, and so on $[1,2]$.

In this paper, we consider the oscillation of the following $m$ th-order forced nonlinear difference equation of the form

$$
\Delta^{m}[x(n)-p(n) x(n-\tau)]+q_{1}(n) \Phi_{\alpha}\left(n-\sigma_{1}\right)+q_{2}(n) \Phi_{\beta}\left(n-\sigma_{2}\right)=f(n),
$$

where $m \geq 1, \tau, \sigma_{1}$ and $\sigma_{2}$ are integers, $\Phi_{*}(u)=|u|^{n-1} u, p(n), q_{1}(n), q_{2}(n)$ and $f(n)$ are real sequences defined on $N=\{0,1,2, \ldots\}$ with $p(n)>0,0<\alpha<1<\beta$ are constants, and

$$
\Delta x(n)=x(n+1)-x(n), \quad \Delta^{i} x(n)=\Delta\left(\Delta^{i-1} x(n)\right), \quad 2 \leq i \leq m .
$$

As usual, a solution of Eq. (1) is said to be oscillatory, if for every integer $N \geq 0$, there exists $n \geq N$ such that $x(n) x(n+1) \leq 0$; otherwise, it is called nonoscillatory.

For the continuous version of Eq. (1), many authors have studied its oscillation (see monograph [3] and references therein). To the best of our knowledge, little has been

(c) 2012 Gao et al.; licensee Springer. This is an Open Access article distributed under the terms of the Creative Commons Attribution License (http://creativecommons.org/licenses/by/2.0), which permits unrestricted use, distribution, and reproduction in any medium, provided the original work is properly cited. 
known about the forced oscillation of Eq. (1) with positive and negative coefficients ( $q_{1} \leq 0$, $q_{2}>0$ or $\left.q_{1} \geq 0, q_{2}<0\right)$ and mixed nonlinearities $(0<\alpha<1, \beta>1)$. For some particular cases of Eq. (1), there have been many oscillation results in [4-19], to name a few. Motivated by the work in [20-22], we study the forced oscillation of Eq. (1) in this paper.

The main contribution of this paper is that we establish some new oscillation criteria for Eq. (1) with positive and negative coefficients and mixed nonlinearities. Unlike some existing results in the literature, all possible values of delays $\tau, \sigma_{1}$ and $\sigma_{2}$ are considered.

\section{Main results}

Throughout this paper, we denote

$$
\begin{aligned}
& \phi_{0}(n, s)=\phi(n, s)=(n-s)^{(k)}=(n-s)(n-s+1) \cdots(n-s+k-1), \quad k \geq m, \\
& \phi_{i}(n, s)=(-1)^{i} \Delta_{s}^{i} \phi(n, s)=C_{k}^{i}(n-s)^{(k-i)}, \quad i=1,2, \ldots, m .
\end{aligned}
$$

By the straightforward computation, it is not difficult to see that

$$
\begin{cases}\phi(n, s)=0, & n \leq s \leq n+k-1, \\ \phi_{i}(n+i+1, n+m)=0, & i=0,1,2, \ldots, m-1, \\ \phi_{m}(n, s) \geq 0, & 0 \leq s \leq n-1,\end{cases}
$$

and

$$
\lim _{n \rightarrow \infty} \frac{\phi_{i}\left(n+i+1, n_{0}\right)}{\phi\left(n+1, n_{0}\right)}=o(1), \quad i=1,2, \ldots, m,
$$

where $n_{0} \geq 0$ is an integer. We also denote $\sum_{s=l}^{k}=0$ if $k<l$.

The following two facts can be easily proved.

Fact 1. Set $F(x)=a x-b x^{\lambda}$, where $x \geq 0, a \geq 0$ and $b>0$. If $\lambda>1, F(x)$ obtains its maxi$\operatorname{mum} F_{\max }=(\lambda-1) \lambda \frac{\lambda}{1-\lambda} a^{\frac{\lambda}{\lambda-1}} b^{\frac{1}{1-\lambda}}$.

Fact 2. Set $G(x)=c x-d x^{\lambda}$, where $x \geq 0, c>0$ and $d \geq 0$. If $0<\lambda<1, G(x)$ obtains its minimum $G_{\min }=(\lambda-1) \lambda \frac{\lambda}{1-\lambda} c^{\frac{\lambda}{\lambda-1}} d \frac{1}{1-\lambda}$.

We now present the main results of this paper as follows.

Theorem 1 Assume that $q_{1}(n) \leq 0, q_{2}(n)>0, \sigma_{1} \geq-m$ and $\sigma_{2}-\tau \leq-m$. If

$$
\begin{aligned}
& \lim _{n \rightarrow \infty} \sup \frac{1}{\phi\left(n+1, n_{0}\right)}\left\{\sum_{s=n_{0}}^{n+m-1}\left[\phi(n, s) f(s)-Q_{2}(n, s)\right]+\sum_{s=n_{0}}^{n-\sigma_{1}-1} Q_{1}(n, s)\right\}=+\infty, \\
& \lim _{n \rightarrow \infty} \inf \frac{1}{\phi\left(n+1, n_{0}\right)}\left\{\sum_{s=n_{0}}^{n+m-1}\left[\phi(n, s) f(s)+Q_{2}(n, s)\right]-\sum_{s=n_{0}}^{n-\sigma_{1}-1} Q_{1}(n, s)\right\}=-\infty,
\end{aligned}
$$

where

$$
\begin{aligned}
& Q_{1}(n, s)=(\alpha-1) \alpha^{\frac{\alpha}{1-\alpha}}\left[\phi_{m}(n+m, s)\right]^{\frac{\alpha}{\alpha-1}}\left[\phi\left(n, s+\sigma_{1}\right)\left|q_{1}\left(s+\sigma_{1}\right)\right|\right]^{\frac{1}{1-\alpha}}, \\
& Q_{2}(n, s)=(\beta-1) \beta^{\frac{\beta}{1-\beta}}\left[\phi_{m}(n+m, s) p(s)\right]^{\frac{\beta}{\beta-1}}\left[\phi\left(n, s+\sigma_{2}-\tau\right) q_{2}\left(s+\sigma_{2}-\tau\right)\right]^{\frac{1}{1-\beta}},
\end{aligned}
$$

all solutions of Eq. (1) are oscillatory. 
Proof Assume to the contrary that there exists a nontrivial solution $x(n)$ of Eq. (1) such that $x(n)$ is nonoscillatory. That is, $x(n)$ does not change sign eventually. Without loss of generality, let $x\left(n-\sigma_{1}\right) \geq 0, x\left(n-\sigma_{2}\right) \geq 0, x(n-\tau) \geq 0$ for $n \geq n_{0}$, where $n_{0} \geq 0$ is sufficiently large. By the straightforward computation, we have

$$
F_{1}(n, s)-F_{2}(n, s)=\sum_{s=n_{0}}^{n+m-1} \phi(n, s) f(s)
$$

where

$$
\begin{aligned}
& F_{1}(n, s)=\sum_{s=n_{0}}^{n+m-1} \phi(n, s) \Delta^{m} x(s)-\sum_{s=n_{0}}^{n+m-1} \phi(n, s)\left|q_{1}(s)\right| x^{\alpha}\left(s-\sigma_{1}\right), \\
& F_{2}(n, s)=\sum_{s=n_{0}}^{n+m-1} \phi(n, s) \Delta^{m}[p(s) x(n-\tau)]-\sum_{s=n_{0}}^{n+m-1} \phi(n, s) q_{2}(s) x^{\beta}\left(s-\sigma_{2}\right) .
\end{aligned}
$$

Noting that

$$
\begin{aligned}
\phi(n, s) \Delta x(s) & =\Delta[\phi(n, s-1) x(s)]+\phi_{1}(n, s-1) x(s) \\
& =\Delta[\phi(n+1, s) x(s)]+\phi_{1}(n+1, s) x(s),
\end{aligned}
$$

we can get from (2), (3) and (4) that

$$
\begin{aligned}
\sum_{s=n_{0}}^{n+m-1} \phi(n, s) \Delta^{m} x(s)= & \sum_{s=n_{0}}^{n+m-1} \Delta\left[\phi(n+1, s) \Delta^{m-1} x(s)\right]+\sum_{s=n_{0}}^{n+m-1} \phi_{1}(n+1, s) \Delta^{m-1} x(s) \\
= & -\phi\left(n+1, n_{0}\right) \Delta^{m-1} x\left(n_{0}\right)+\sum_{s=n_{0}}^{n+m-1} \phi_{1}(n+1, s) \Delta^{m-1} x(s) \\
= & -\phi\left(n+1, n_{0}\right) \Delta^{m-1} x\left(n_{0}\right)+\sum_{s=n_{0}}^{n+m-1} \Delta\left[\phi_{1}(n+2, s) \Delta^{m-2} x(s)\right] \\
& +\sum_{s=n_{0}}^{n+m-1} \phi_{2}(n+2, s) \Delta^{m-2} x(s) \\
= & -\phi\left(n+1, n_{0}\right) \Delta^{m-1} x\left(n_{0}\right)-\phi_{1}\left(n+2, n_{0}\right) \Delta^{m-2} x\left(n_{0}\right) \\
& +\sum_{s=n_{0}}^{n+m-1} \phi_{2}(n+2, s) \Delta^{m-2} x(s) \\
= & -\sum_{i=0}^{m-1} \phi_{i}\left(n+i+1, n_{0}\right) \Delta^{m-i-1} x\left(n_{0}\right)+\sum_{s=n_{0}}^{n+m-1} \phi_{m}(n+m, s) x(s) .
\end{aligned}
$$

Since $\phi(n, s)=0$ for $n \leq s \leq n+m-1$ due to (4), we get from (11) that

$$
\begin{aligned}
F_{1}(n, s)= & \sum_{s=n_{0}}^{n+m-1} \phi_{m}(n+m, s) x(s)-\sum_{s=n_{0}}^{n-1} \phi(n, s)\left|q_{1}(s)\right| x^{\alpha}\left(s-\sigma_{1}\right) \\
& -\sum_{i=0}^{m-1} \phi_{i}\left(n+i+1, n_{0}\right) \Delta^{m-i-1} x\left(n_{0}\right)
\end{aligned}
$$




$$
\begin{aligned}
= & \sum_{s=n_{0}}^{n+m-1} \phi_{m}(n+m, s) x(s)-\sum_{s=n_{0}-\sigma_{1}}^{n-\sigma_{1}-1} \phi\left(n, s+\sigma_{1}\right)\left|q_{1}\left(s+\sigma_{1}\right)\right| x^{\alpha}(s) \\
& -\sum_{i=0}^{m-1} \phi_{i}\left(n+i+1, n_{0}\right) \Delta^{m-i-1} x\left(n_{0}\right) .
\end{aligned}
$$

Noting that $\sigma_{1} \geq-m$, we have that $n+m-1 \geq n-\sigma_{1}-1$. Therefore, we get from (12) that

$$
\begin{aligned}
F_{1}(n, s) \geq & \sum_{s=n_{0}}^{n-\sigma_{1}-1}\left[\phi_{m}(n+m, s) x(s)-\phi\left(n, s+\sigma_{1}\right)\left|q_{1}\left(s+\sigma_{1}\right)\right| x^{\alpha}(s)\right] \\
& -\sum_{s=n_{0}-\sigma_{1}}^{n_{0}-1} \phi\left(n, s+\sigma_{1}\right)\left|q_{1}\left(s+\sigma_{1}\right)\right| x^{\alpha}(s) \\
& -\sum_{i=0}^{m-1} \phi_{i}\left(n+i+1, n_{0}\right) \Delta^{m-i-1} x\left(n_{0}\right) .
\end{aligned}
$$

By Fact 2 and (13), it is not difficult to see that

$$
\begin{aligned}
F_{1}(n, s) \geq & \sum_{s=n_{0}}^{n-\sigma_{1}-1} Q_{1}(n, s)-\sum_{s=n_{0}-\sigma_{1}}^{n_{0}-1} \phi\left(n, s+\sigma_{1}\right)\left|q_{1}\left(s+\sigma_{1}\right)\right| x^{\alpha}(s) \\
& -\sum_{i=0}^{m-1} \phi_{i}\left(n+i+1, n_{0}\right) \Delta^{m-i-1} x\left(n_{0}\right),
\end{aligned}
$$

where $Q_{1}(n, s)$ is defined by $(8)$.

On the other hand, similar to the above analysis, we have that

$$
\begin{aligned}
F_{2}(n, s)= & \sum_{s=n_{0}}^{n+m-1} \phi_{m}(n+m, s) p(s) x(s-\tau)-\sum_{s=n_{0}}^{n+m-1} \phi(n, s) q_{2}(s) x^{\beta}\left(s-\sigma_{2}\right) \\
& -\sum_{i=0}^{m-1} \phi_{i}\left(n+i+1, n_{0}\right) \Delta^{m-i-1}\left[p\left(n_{0}\right) x\left(n_{0}-\tau\right)\right] \\
= & \sum_{s=n_{0}}^{n+m-1} \phi_{m}(n+m, s) p(s) x(s-\tau)-\sum_{s=n_{0}}^{n-1} \phi(n, s) q_{2}(s) x^{\beta}\left(s-\sigma_{2}\right) \\
& -\sum_{i=0}^{m-1} \phi_{i}\left(n+i+1, n_{0}\right) \Delta^{m-i-1}\left[x\left(n_{0}-\tau\right) p\left(n_{0}\right)\right] \\
= & \sum_{s=n_{0}}^{n+m-1} \phi_{m}(n+m, s) p(s) x(s-\tau) \\
& -\sum_{s=n_{0}-\sigma_{2}+\tau}^{n-\sigma_{2}+\tau-1} \phi\left(n, s+\sigma_{2}-\tau\right) q_{2}\left(s+\sigma_{2}-\tau\right) x^{\beta}(s-\tau) \\
& -\sum_{i=0}^{m-1} \phi_{i}\left(n+i+1, n_{0}\right) \Delta^{m-i-1}\left[p\left(n_{0}\right) x\left(n_{0}-\tau\right)\right] .
\end{aligned}
$$


Since $\sigma_{2}-\tau \leq-m$, we have that $n-\sigma_{2}+\tau-1 \geq n+m-1$. By (15), we get

$$
\begin{aligned}
F_{2}(n, s) \leq & \sum_{s=n_{0}}^{n+m-1}\left[\phi_{m}(n+m, s) p(s) x(s-\tau)-\phi\left(n, s+\sigma_{2}-\tau\right) q_{2}\left(s+\sigma_{2}-\tau\right) x^{\beta}(s-\tau)\right] \\
& +\sum_{s=n_{0}}^{n_{0}-\sigma_{2}+\tau-1} \phi\left(n, s+\sigma_{2}-\tau\right) q_{2}\left(s+\sigma_{2}-\tau\right) x^{\beta}(s-\tau) \\
& -\sum_{i=0}^{m-1} \phi_{i}\left(n+i+1, n_{0}\right) \Delta^{m-i-1}\left[p\left(n_{0}\right) x\left(n_{0}-\tau\right)\right] .
\end{aligned}
$$

By Fact 1 and (16), we have that

$$
\begin{aligned}
F_{2}(n, s) \leq & \sum_{s=n_{0}}^{n+m-1} Q_{2}(n, s)+\sum_{s=n_{0}}^{n_{0}-\sigma_{2}+\tau-1} \phi\left(n, s+\sigma_{2}-\tau\right) q_{2}\left(s+\sigma_{2}-\tau\right) x^{\beta}(s-\tau) \\
& -\sum_{i=0}^{m-1} \phi_{i}\left(n+i+1, n_{0}\right) \Delta^{m-i-1}\left[p\left(n_{0}\right) x\left(n_{0}-\tau\right)\right]
\end{aligned}
$$

where $Q_{2}(n, s)$ is defined by (9).

Multiplying $\frac{1}{\phi\left(n+1, n_{0}\right)}$ on both sides of (10), by (14), (17) and (5), we have that there exists a constant $M_{1}$ such that

$$
\frac{1}{\phi\left(n+1, n_{0}\right)}\left\{\sum_{s=n_{0}}^{n+m-1}\left[\phi(n, s) f(s)+Q_{2}(n, s)\right]-\sum_{s=n_{0}}^{n-\sigma_{1}-1} Q_{1}(n, s)\right\} \geq M_{1}
$$

which contradicts (7). For the case when $x(n)$ is eventually negative, we can similarly get a contradiction to (6). This completes the proof of Theorem 1.

Theorem 2 Assume that $q_{1}(n) \geq 0, q_{2}(n)<0, \sigma_{1}-\tau \geq-m$ and $\sigma_{2} \leq-m$. If

$$
\begin{aligned}
& \lim _{n \rightarrow \infty} \sup \frac{1}{\phi\left(n+1, n_{0}\right)}\left\{\sum_{s=n_{0}}^{n+m-1}\left[\phi(n, s) f(s)-P_{1}(n, s)\right]+\sum_{s=n_{0}}^{n-\sigma_{2}+\tau-1} P_{2}(n, s)\right\}=+\infty, \\
& \lim _{n \rightarrow \infty} \inf \frac{1}{\phi\left(n+1, n_{0}\right)}\left\{\sum_{s=n_{0}}^{n+m-1}\left[\phi(n, s) f(s)+P_{1}(n, s)\right]-\sum_{s=n_{0}}^{n-\sigma_{2}+\tau-1} P_{2}(n, s)\right\}=-\infty,
\end{aligned}
$$

where

$$
\begin{aligned}
& P_{1}(n, s)=(\beta-1) \beta^{\frac{\beta}{1-\beta}}\left[\phi_{m}(n+m, s)\right]^{\frac{\beta}{\beta-1}}\left[\phi\left(n, s+\sigma_{2}\right)\left|q_{2}\left(s+\sigma_{2}\right)\right|\right]^{\frac{1}{1-\beta}}, \\
& P_{2}(n, s)=(\alpha-1) \alpha^{\frac{\alpha}{1-\alpha}}\left[\phi_{m}(n+m, s) p(s)\right]^{\frac{\alpha}{\alpha-1}}\left[\phi\left(n, s+\sigma_{1}-\tau\right) q_{1}\left(s+\sigma_{1}-\tau\right)\right]^{\frac{1}{1-\alpha}},
\end{aligned}
$$

all solutions of Eq. (1) are oscillatory.

Proof Suppose to the contrary that there exists a nontrivial solution $x(n)$ of Eq. (1) such that $x(n)$ is nonoscillatory. We may let $x\left(n-\sigma_{1}\right) \geq 0, x\left(n-\sigma_{2}\right) \geq 0, x(n-\tau) \geq 0$ for $n \geq n_{0}$, 
where $n_{0} \geq 0$ is sufficiently large. By the straightforward computation, we get from Eq. (1) that

$$
G_{1}(n, s)-G_{2}(n, s)=\sum_{s=n_{0}}^{n+m-1} \phi(n, s) f(s),
$$

where

$$
\begin{aligned}
& G_{1}(n, s)=\sum_{s=n_{0}}^{n+m-1} \phi(n, s) \Delta^{m} x(s)-\sum_{s=n_{0}}^{n+m-1} \phi(n, s)\left|q_{2}(s)\right| x^{\beta}\left(s-\sigma_{2}\right), \\
& G_{2}(n, s)=\sum_{s=n_{0}}^{n+m-1} \phi(n, s) \Delta^{m}[p(s) x(n-\tau)]-\sum_{s=n_{0}}^{n+m-1} \phi(n, s) q_{1}(s) x^{\alpha}\left(s-\sigma_{1}\right) .
\end{aligned}
$$

Noticing that $\phi(n, s)=0$ for $n \leq s \leq n+m-1$, we get from (11) that

$$
\begin{aligned}
G_{1}(n, s)= & \sum_{s=n_{0}}^{n+m-1} \phi_{m}(n+m, s) x(s)-\sum_{s=n_{0}}^{n-1} \phi(n, s)\left|q_{2}(s)\right| x^{\beta}\left(s-\sigma_{2}\right) \\
& -\sum_{i=0}^{m-1} \phi_{i}\left(n+i+1, n_{0}\right) \Delta^{m-i-1} x\left(n_{0}\right) \\
= & \sum_{s=n_{0}}^{n+m-1} \phi_{m}(n+m, s) x(s)-\sum_{s=n_{0}-\sigma_{2}}^{n-\sigma_{2}-1} \phi\left(n, s+\sigma_{2}\right)\left|q_{2}\left(s+\sigma_{2}\right)\right| x^{\beta}(s) \\
& -\sum_{i=0}^{m-1} \phi_{i}\left(n+i+1, n_{0}\right) \Delta^{m-i-1} x\left(n_{0}\right) .
\end{aligned}
$$

Since $\sigma_{2} \leq-m$, we have that $n+m-1 \leq n-\sigma_{2}-1$. Thus, we can get from (23) that

$$
\begin{aligned}
G_{1}(n, s) \leq & \sum_{s=n_{0}}^{n+m-1}\left[\phi_{m}(n+m, s) x(s)-\phi\left(n, s+\sigma_{2}\right)\left|q_{2}\left(s+\sigma_{2}\right)\right| x^{\beta}(s)\right] \\
& +\sum_{s=n_{0}}^{n_{0}-\sigma_{2}-1} \phi\left(n, s+\sigma_{2}\right)\left|q_{2}\left(s+\sigma_{2}\right)\right| x^{\beta}(s) \\
& -\sum_{i=0}^{m-1} \phi_{i}\left(n+i+1, n_{0}\right) \Delta^{m-i-1} x\left(n_{0}\right) .
\end{aligned}
$$

By Fact 1 and (24), it is easy to see that

$$
\begin{aligned}
G_{1}(n, s) \leq & \sum_{s=n_{0}}^{n+m-1} P_{1}(n, s)+\sum_{s=n_{0}}^{n_{0}-\sigma_{2}-1} \phi\left(n, s+\sigma_{2}\right)\left|q_{2}\left(s+\sigma_{2}\right)\right| x^{\beta}(s) \\
& -\sum_{i=0}^{m-1} \phi_{i}\left(n+i+1, n_{0}\right) \Delta^{m-i-1} x\left(n_{0}\right),
\end{aligned}
$$

where $P_{1}(n, s)$ is defined by $(20)$. 
On the other hand, similar to the computation of (11), we can get

$$
\begin{aligned}
G_{2}(n, s)= & \sum_{s=n_{0}}^{n+m-1} \phi_{m}(n+m, s) p(s) x(s-\tau)-\sum_{s=n_{0}}^{n-1} \phi(n, s) q_{1}(s) x^{\alpha}\left(s-\sigma_{1}\right) \\
& -\sum_{i=0}^{m-1} \phi_{i}\left(n+i+1, n_{0}\right) \Delta^{m-i-1}\left[p\left(n_{0}\right) x\left(n_{0}-\tau\right)\right] \\
= & \sum_{s=n_{0}}^{n+m-1} \phi_{m}(n+m, s) p(s) x(s-\tau) \\
& -\sum_{s=n_{0}-\sigma_{1}+\tau}^{n-\sigma_{1}+\tau-1} \phi\left(n, s+\sigma_{1}-\tau\right) q_{1}\left(s+\sigma_{1}-\tau\right) x^{\alpha}(s-\tau) \\
& -\sum_{i=0}^{m-1} \phi_{i}\left(n+i+1, n_{0}\right) \Delta^{m-i-1}\left[p\left(n_{0}\right) x\left(n_{0}-\tau\right)\right] .
\end{aligned}
$$

Noting that $\sigma_{1}-\tau \geq-m$ implies $n+m-1 \geq n-\sigma_{1}+\tau-1$, we get from (26) that

$$
\begin{aligned}
G_{2}(n, s) \geq & \sum_{s=n_{0}}^{n-\sigma_{1}+\tau-1}\left[\phi_{m}(n+m, s) p(s) x(s-\tau)-\phi\left(n, s+\sigma_{1}-\tau\right) q_{1}\left(s+\sigma_{1}-\tau\right) x^{\alpha}(s-\tau)\right] \\
& -\sum_{s=n_{0}-\sigma_{1}+\tau}^{n_{0}-1} \phi\left(n, s+\sigma_{1}-\tau\right) q_{1}\left(s+\sigma_{1}-\tau\right) x^{\alpha}(s-\tau) \\
& -\sum_{i=0}^{m-1} \phi_{i}\left(n+i+1, n_{0}\right) \Delta^{m-i-1}\left[p\left(n_{0}\right) x\left(n_{0}-\tau\right)\right]
\end{aligned}
$$

By Fact 2 and (27), we have that

$$
\begin{aligned}
G_{2}(n, s) \geq & \sum_{s=n_{0}}^{n-\sigma_{1}+\tau-1} P_{2}(n, s)-\sum_{s=n_{0}-\sigma_{1}+\tau}^{n_{0}-1} \phi\left(n, s+\sigma_{1}-\tau\right) q_{1}\left(s+\sigma_{1}-\tau\right) x^{\alpha}(s-\tau) \\
& -\sum_{i=0}^{m-1} \phi_{i}\left(n+i+1, n_{0}\right) \Delta^{m-i-1}\left[p\left(n_{0}\right) x\left(n_{0}-\tau\right)\right],
\end{aligned}
$$

where $P_{2}(n, s)$ is defined by $(21)$.

Multiplying $\frac{1}{\phi\left(n+1, n_{0}\right)}$ on both sides of (22), from (25), (28) and (5), we have that there exists a constant $M_{2}$ such that

$$
\frac{1}{\phi\left(n+1, n_{0}\right)}\left\{\sum_{s=n_{0}}^{n+m-1}\left[\phi(n, s) f(s)-P_{1}(n, s)\right]+\sum_{s=n_{0}}^{n-\sigma_{2}+\tau-1} P_{2}(n, s)\right\} \leq M_{2} .
$$

This is a contradiction to (18). For the case when $x(n)$ is eventually negative, we can similarly get a contradiction to (19). This completes the proof of Theorem 2.

By Theorems 1 and 2, the following two corollaries are immediate. 
Corollary 1 Assume that $q_{1}(n) \geq 0, q_{2}(n)>0$ and $\sigma_{2}-\tau \leq-m$. If

$$
\begin{aligned}
& \lim _{n \rightarrow \infty} \sup \frac{1}{\phi\left(n+1, n_{0}\right)} \sum_{s=n_{0}}^{n+m-1}\left[\phi(n, s) f(s)-Q_{2}(n, s)\right]=+\infty, \\
& \lim _{n \rightarrow \infty} \inf \frac{1}{\phi\left(n+1, n_{0}\right)} \sum_{s=n_{0}}^{n+m-1}\left[\phi(n, s) f(s)+Q_{2}(n, s)\right]=-\infty,
\end{aligned}
$$

where $Q_{2}(n, s)$ is defined by (9), all solutions of Eq. (1) are oscillatory for any constant $\sigma_{1}$.

Proof In fact, we have that $F_{1}(n, s) \geq 0$ for any constant $\sigma_{1}$ since $q_{1}(n, s) \geq 0$. So, we can drop $F_{1}(n, s)$ in the estimation of $(10)$. The other proof runs as that of Theorem 1 , and hence it is omitted.

Corollary 2 Assume that $q_{1}(n) \leq 0, q_{2}(n)<0$ and $\sigma_{2} \leq-m$. If

$$
\begin{aligned}
& \lim _{n \rightarrow \infty} \sup \frac{1}{\phi\left(n+1, n_{0}\right)} \sum_{s=n_{0}}^{n+m-1}\left[\phi(n, s) f(s)-P_{1}(n, s)\right]=+\infty, \\
& \lim _{n \rightarrow \infty} \inf \frac{1}{\phi\left(n+1, n_{0}\right)} \sum_{s=n_{0}}^{n+m-1}\left[\phi(n, s) f(s)+P_{1}(n, s)\right]=-\infty
\end{aligned}
$$

where $P_{1}(n, s)$ is defined by (20), all nontrivial solutions of Eq. (1) are oscillatory.

Proof For this case, we have that $G_{2}(n, s) \geq 0$ for any constant $\sigma_{1}$ since $q_{1}(n, s) \leq 0$. Therefore, we can drop $G_{2}(n, s)$ in the estimation of (22). The other proof runs as that of Theorem 2 .

For other cases of $\sigma_{1}$ and $\sigma_{2}$ that are not covered by Theorem 1 and Theorem 2, the above method usually does not give sufficient conditions for the oscillation of all solutions of Eq. (1). However, when assuming that the solutions of Eq. (1) satisfy appropriate conditions, sufficient conditions for such solutions can also be derived. In the following, we are focused on the oscillation of all solutions of Eq. (1) satisfying $x(n)=O\left(n^{r}\right)$ for some $r>0$. Here, $x(n)=O\left(n^{r}\right)$ means that there exists a constant $c>0$ such that $|x(n)| \leq c n^{r}$ for $n \geq n_{0}$.

Theorem 3 Assume that $q_{1}(n) \leq 0, q_{2}(n)>0$, and (6) and (7) hold. All solutions satisfying $x(n)=O\left(n^{r}\right)$ are oscillatory if one of the following conditions holds:

(i) $\sigma_{1}<-m, \sigma_{2}-\tau \leq-m$, and

$$
\lim _{n \rightarrow \infty} \sup \frac{1}{\phi\left(n+1, n_{0}\right)} \sum_{s=n+m}^{n-\sigma-1}\left[\phi_{m}(n+m, s) s^{r}\right]<\infty
$$

(ii) $\sigma_{1} \geq-m, \sigma_{2}-\tau>-m$, and

$$
\lim _{n \rightarrow \infty} \sup \frac{1}{\phi\left(n+1, n_{0}\right)} \sum_{s=n-\sigma_{2}+\tau}^{n+m-1}\left[\phi\left(n, s+\sigma_{2}-\tau\right) q_{2}\left(s+\sigma_{2}-\tau\right) s^{r \beta}\right]<\infty
$$

(iii) $\sigma_{1}<-m, \sigma_{2}-\tau>-m$, (29) and (30) hold. 
Proof Assume that there exists a nontrivial solution $x(n)$ of Eq. (1) such that $x(n)$ is nonoscillatory. Without loss of generality, let $x\left(n-\sigma_{1}\right) \geq 0, x\left(n-\sigma_{2}\right) \geq 0, x(n-\tau) \geq 0$ for $n \geq n_{0}$, where $n_{0} \geq 0$ is sufficiently large.

(i) For the case $\sigma_{1}<-m$, we have that $n+m-1<n-\sigma_{1}-1$. Therefore, we get from (12) that

$$
\begin{aligned}
F_{1}(n, s)= & \sum_{s=n_{0}}^{n-\sigma_{1}-1}\left[\phi_{m}(n+m, s) x(s)-\phi\left(n, s+\sigma_{1}\right)\left|q_{1}\left(s+\sigma_{1}\right)\right| x^{\alpha}(s)\right] \\
& -\sum_{s=n+m}^{n-\sigma_{1}-1} \phi_{m}(n+m, s) x(s)+\sum_{s=n_{0}}^{n_{0}-\sigma_{1}-1} \phi\left(n, s+\sigma_{1}\right)\left|q_{1}\left(s+\sigma_{1}\right)\right| x^{\alpha}(s) \\
& -\sum_{i=0}^{m-1} \phi_{i}\left(n+i+1, n_{0}\right) \Delta^{m-i-1} x\left(n_{0}\right) .
\end{aligned}
$$

By Fact 2 and (31), and noting that $x(n) \leq c n^{r}$ for $n \geq n_{0}$ and some constant $c>0$, we get

$$
\begin{aligned}
F_{1}(n, s) \geq & \sum_{s=n_{0}}^{n-\sigma_{1}-1} Q_{1}(n, s)-c \sum_{s=n+m}^{n-\sigma_{1}-1} \phi\left(n, s+\sigma_{1}\right)\left|q_{1}\left(s+\sigma_{1}\right)\right| s^{r} \\
& -\sum_{i=0}^{m-1} \phi_{i}\left(n+i+1, n_{0}\right) \Delta^{m-i-1} x\left(n_{0}\right),
\end{aligned}
$$

where $Q_{1}(n, s)$ is defined by (8). Multiplying $\frac{1}{\phi\left(n+1, n_{0}\right)}$ on both sides of (10), from (32), (17), (5) and (29), we get a contradiction to (7).

(ii) For the case $\sigma_{2}-\tau>-m$, we have that $n-\sigma_{2}+\tau-1<n+m-1$. By (15), we get

$$
\begin{aligned}
F_{2}(n, s)= & \sum_{s=n_{0}}^{n+m-1}\left[\phi_{m}(n+m, s) p(s) x(s-\tau)-\phi\left(n, s+\sigma_{2}-\tau\right) q_{2}\left(s+\sigma_{2}-\tau\right) x^{\beta}(s-\tau)\right] \\
& +\sum_{s=n-\sigma_{2}+\tau}^{n+m-1} \phi\left(n, s+\sigma_{2}-\tau\right) q_{2}\left(s+\sigma_{2}-\tau\right) x^{\beta}(s-\tau) \\
& -\sum_{s=n_{0}-\sigma_{2}+\tau}^{n_{0}-1} \phi\left(n, s+\sigma_{2}-\tau\right) q_{2}\left(s+\sigma_{2}-\tau\right) x^{\beta}(s-\tau) \\
& -\sum_{i=0}^{m-1} \phi_{i}\left(n+i+1, n_{0}\right) \Delta^{m-i-1}\left[p\left(n_{0}\right) x\left(n_{0}-\tau\right)\right] .
\end{aligned}
$$

By Fact 1 and (33), and noting that $x(n) \leq c n^{r}$ for $n \geq n_{0}$, we have that

$$
\begin{aligned}
F_{2}(n, s) \leq & \sum_{s=n_{0}}^{n+m-1} Q_{2}(n, s)+c^{\beta} \sum_{s=n-\sigma_{2}+\tau}^{n+m-1} \phi\left(n, s+\sigma_{2}-\tau\right) q_{2}\left(s+\sigma_{2}-\tau\right)(s-\tau)^{\beta r} \\
& -\sum_{i=0}^{m-1} \phi_{i}\left(n+i+1, n_{0}\right) \Delta^{m-i-1}\left[p\left(n_{0}\right) x\left(n_{0}-\tau\right)\right],
\end{aligned}
$$

where $Q_{2}(n, s)$ is defined by (9). Multiplying $\frac{1}{\phi\left(n+1, n_{0}\right)}$ on both sides of (10), from (14), (34) and (5), we can get a contradiction to (7). 
(iii) Multiplying $\frac{1}{\phi\left(n+1, n_{0}\right)}$ on both sides of (10), from (32), (34), (29) and (30), we derive a contradiction. The proof of Theorem 3 is complete.

Theorem 4 Assume that $q_{1}(n) \geq 0, q_{2}(n)<0$, (18) and (19) hold. All solutions satisfying $x(n)=O\left(n^{r}\right)$ are oscillatory if one of the following conditions holds:

(i) $\sigma_{1}-\tau \geq-m, \sigma_{2}>-m$, and

$$
\lim _{n \rightarrow \infty} \sup \frac{1}{\phi\left(n+1, n_{0}\right)} \sum_{s=n-\sigma_{2}}^{n+m-1}\left[\phi\left(n, s+\sigma_{2}\right)\left|q_{2}\left(s+\sigma_{2}\right)\right| s^{r \beta}\right]<\infty
$$

(ii) $\sigma_{1}-\tau \leq-m, \sigma_{2} \leq-m$, and

$$
\lim _{n \rightarrow \infty} \sup \frac{1}{\phi\left(n+1, n_{0}\right)} \sum_{s=n+m}^{n-\sigma_{1}+\tau-1}\left[\phi_{m}(n+m, s) p(s)(s-\tau)^{r}\right]<\infty
$$

(iii) $\sigma_{1}-\tau<-m, \sigma_{2}>-m$, (35) and (36) hold.

Proof The proof is similar to that of Theorem 2 and Theorem 3, and hence it is omitted.

\section{Examples}

We here work out two simple examples to illustrate the importance of Theorem 1 and Theorem 2.

Example 1 Consider the following third-order neutral difference equation:

$$
\Delta^{3}[x(n)-x(n-1)]-\Phi_{1 / 2}\left(n-\sigma_{1}\right)+\Phi_{2}\left(n-\sigma_{2}\right)=n^{k} \sin n, \quad n \geq 0,
$$

where $k>0$ is a constant. It is evident that $m=3, \sigma_{1}=\tau=1, \sigma_{2}=-2, \alpha=1 / 2, \beta=2$, $p(n) \equiv 1, q_{1}(n) \equiv-1, q_{2}(n) \equiv 1$ and $f(n)=n^{k} \sin n$. If we choose $\phi(n, s)=(n-s)^{(3)}$, we have $\phi_{3}(n, s)=6$. By the straightforward computation, we have that

$$
Q_{1}(n, s)=-\left[(n-s-1)^{(2)}\right]^{2} / 24, \quad Q_{2}(n, s)=\left[(n-s+3)^{(3)}\right]^{-1} .
$$

By Theorem 1, we have that every solution of Eq. (37) is oscillatory if

$$
\begin{aligned}
& \lim _{n \rightarrow \infty} \sup \frac{1}{(n+1)(n+2)(n+3)}\left[\sum_{s=0}^{n+2}(n-s)^{(3)} s^{k} \sin s+\sum_{s=0}^{n-2} Q_{1}(n, s)\right]=+\infty \\
& \lim _{n \rightarrow \infty} \inf \frac{1}{(n+1)(n+2)(n+3)}\left[\sum_{s=0}^{n+2}(n-s)^{(3)} s^{k} \sin s-\sum_{s=n_{0}}^{n-2} Q_{1}(n, s)\right]=-\infty
\end{aligned}
$$

It is not difficult to see that the above two inequalities hold for appropriate $k>0$.

Example 2 Consider the following third-order neutral difference equation:

$$
\Delta^{3}[x(n)-x(n-1)]+\Phi_{1 / 2}\left(n-\sigma_{1}\right)-\Phi_{2}\left(n-\sigma_{2}\right)=n^{k} \cos n, \quad n \geq 0,
$$


where $k>0$ is a constant. It is obvious that $m=3, \sigma_{1}=-2, \sigma_{2}=\tau=1, \alpha=1 / 2, \beta=2$, $p(n) \equiv 1, q_{1}(n) \equiv 1, q_{2}(n) \equiv-1$ and $f(n)=n^{k} \cos n$. We also choose $\phi(n, s)=(n-s)^{(3)}$. By the straightforward computation, we have that

$$
P_{1}(n, s)=\left[(n-s+3)^{(3)}\right]^{-1}, \quad P_{2}(n, s)=-\left[(n-s-1)^{(3)}\right]^{2} / 24
$$

By Theorem 2, we have that every solution of Eq. (38) is oscillatory if

$$
\begin{aligned}
& \lim _{n \rightarrow \infty} \sup \frac{1}{(n+1)(n+2)(n+3)}\left[\sum_{s=0}^{n+2}(n-s)^{(3)} s^{k} \cos s+\sum_{s=0}^{n-1} P_{2}(n, s)\right]=+\infty, \\
& \lim _{n \rightarrow \infty} \inf \frac{1}{(n+1)(n+2)(n+3)}\left[\sum_{s=0}^{n+2}(n-s)^{(3)} s^{k} \cos s-\sum_{s=0}^{n-1} P_{2}(n, s)\right]=-\infty
\end{aligned}
$$

It is not difficult to see that the above two inequalities hold for appropriate $k>0$.

\section{Competing interests}

The authors declare that they have no competing interests.

\section{Authors' contributions}

YS framed the problem. YG solved the problem. BZ and HL made necessary changes in the proof of the theorems. All authors read and approved the manuscript.

\section{Acknowledgements}

The authors thank the reviewers for their helpful and valuable comments on this paper. This work was supported by the Natural Science Foundation of Shandong Province (grant nos. ZR2010AL002 and JQ201119) and the National Natural Science Foundation of China (grant no. 61174217).

Received: 22 March 2012 Accepted: 2 July 2012 Published: 19 July 2012

\section{References}

1. Agarwal, RP: Difference Equations and Inequalities: Theory, Methods, and Applications. Dekker, New York (1992)

2. Agarwal, RP, Wong, PJY: Advanced Topics in Difference Equations. Kluwer Academic, Dordrecht (1997)

3. Györi, I, Ladas, G: Oscillation Theory of Delay Differential Equations. Clarendon, Oxford (1991)

4. Sun, YG, Saker, SH: Oscillation for second-order nonlinear neutral delay difference equations. Appl. Math. Comput. 163, 909-918 (2005)

5. Agarwal, RP, Manuel, MMS, Thandapani, E: Oscillatory and nonoscillatory behavior of second-order neutral delay difference equations. Math. Comput. Model. 24, 5-11 (1996)

6. Agarwal, RP, Manuel, MMS, Thandapani, E: Oscillatory and nonoscillatory behavior of second-order neutral delay difference equations II. Appl. Math. Lett. 10, 103-109 (1997)

7. Thandapani, E, Kavitha, N, Pinelas, S: Oscillation criteria for second-order nonlinear neutral difference equations of mixed type. Adv. Differ. Equ. 2012, 4 (2012)

8. Agarwal, RP, Thandapani, E, Wong, PJY: Oscillations of higher-order neutral difference equations. Appl. Math. Lett. 10, 71-78 (1997)

9. Jiang, J: Oscillation of second order nonlinear neutral delay difference equations. Appl. Math. Comput. 146, 791-801 (2003)

10. Lou, JW, Bainov, DD: Oscillatory and asymptotic behavior of second-order neutral difference equations with maximum. J. Comput. Appl. Math. 131, 331-341 (2001)

11. Saker, SH: New oscillation criteria for second-order nonlinear neutral delay difference equations. Appl. Math. Comput. 142, 99-111 (2003)

12. Yildiz, MK, Öcalan, Ö: Oscillation results for higher order nonlinear neutral delay difference equations. Appl. Math. Lett. 20, 243-247 (2007)

13. Liu, B, Yan, J: Oscillation theorems for nonlinear neutral difference equations. J. Differ. Equ. Appl. 1, $307-315$ (1995)

14. Lalli, BS, Zhang, BG, Li, JZ: On the oscillation of solutions and existence of positive solutions of neutral difference equations. J. Math. Anal. Appl. 158, 213-233 (1991)

15. Zafer, A, Dahiya, RS: Oscillation of a neutral difference equations. Appl. Math. Lett. 6, 71-74 (1993)

16. Koo, NJ: Oscillation of neutral difference equations. J. Chungcheong Math. Soc. 12, 1-7 (1999)

17. Chen, MP, Lalli, BS, Yu, JS: Oscillation in neutral difference equations with variable coefficients. Comput. Math. Appl. 29, 5-11 (1995)

18. Yu, JS, Wang, ZC: Asymptotic behavior and oscillation in neutral delay difference equations. Funkc. Ekvacioj 37 , 241-248 (1994) 
19. Tang, $\mathrm{XH}, \mathrm{Yu}, \mathrm{JS}$, Peng, DH: Oscillation and nonoscillation of neutral difference equations with positive and negative coefficients. Comput. Math. Appl. 39, 169-181 (2000)

20. Agarwal, RP, Grace, SR: Forced oscillation of $n$th order nonlinear differential equations. Appl. Math. Lett. 13, 53-57 (2000)

21. Ou, CH, Wong, JSW: Forced oscillation of $n$ th-order functional differential equations. J. Math. Anal. Appl. 262, 722-732 (2001)

22. Yang, X: Forced oscillation of $n$th nonlinear differential equations. Appl. Math. Comput. 134, $301-305$ (2003)

doi:10.1186/1687-1847-2012-110

Cite this article as: Gao et al.: Forced oscillation of higher-order nonlinear neutral difference equations with positive and negative coefficients. Advances in Difference Equations 2012 2012:110.

Submit your manuscript to a SpringerOpen ${ }^{\circ}$ journal and benefit from:

- Convenient online submission

- Rigorous peer review

- Immediate publication on acceptance

- Open access: articles freely available online

- High visibility within the field

- Retaining the copyright to your article

Submit your next manuscript at $>$ springeropen.com 